\title{
Erratum: Molecular-dynamics approach for studying the nonequilibrium behavior of x-ray-heated solid-density matter [Phys. Rev. E 96, 023205 (2017)]
}

Malik Muhammad Abdullah, Anurag, Zoltan Jurek, Sang-Kil Son, and Robin Santra

(Received 20 January 2021; published 4 February 2021)

DOI: 10.1103/PhysRevE.103.029901

Equation (2) in the original paper was incomplete. The expression actually used for creating Fig. 2 reads

$$
\begin{aligned}
E(T)= & \sum_{p} \varepsilon_{p} \tilde{n}_{p}(\mu, T) \int_{r \leqslant r_{s}} d^{3} r\left|\psi_{p}(\mathbf{r})\right|^{2} \\
& -\frac{1}{2} \int_{r \leqslant r_{s}} d^{3} r \int_{r^{\prime} \leqslant r_{s}} d^{3} r^{\prime} \frac{\rho(\mathbf{r}, T) \rho\left(\mathbf{r}^{\prime}, T\right)}{\left|\mathbf{r}-\mathbf{r}^{\prime}\right|}+\frac{3}{8}\left(\frac{3}{\pi}\right)^{1 / 3} \int_{r \leqslant r_{s}} d^{3} r \rho(\mathbf{r}, T)^{4 / 3},
\end{aligned}
$$

where $\rho(\mathbf{r}, T)=\sum_{p}\left|\psi_{p}(\mathbf{r})\right|^{2} \tilde{n}_{p}(\mu, T)$ is the electron density.

Figure 3 in the original paper showed the kinetic-energy distribution of the electron plasma for (a) low fluence, (c) medium fluence, and (e) high fluence, together with the curve fitted to the Maxwell-Boltzmann distribution. The Maxwell-Boltzmann distribution for the energy reads

$$
f_{E}(E)=2 \sqrt{\frac{E}{\pi T^{3}}} e^{-E / T} .
$$

However, the curves in Fig. 3 were fitted to a wrong formula,

$$
f_{\text {wrong }}(E)=\frac{E}{T^{2}} e^{-E / T} .
$$

Therefore, Figs. 3(a), 3(c), and 3(e) should be replotted as shown here. The fitted curves look alike, but the fitted temperatures become higher than before. In Table I, the XMDYN temperature should be $10 \mathrm{eV}$ for low fluence, $86 \mathrm{eV}$ for medium fluence, and $135 \mathrm{eV}$ for high fluence. Note that the correct expression was already used in Fig. 8.

These corrections have no consequence for the conclusions in our original paper.

The authors are indebted to Dr. R. Jin for his investigation of the fitting procedure underlying Fig. 3.
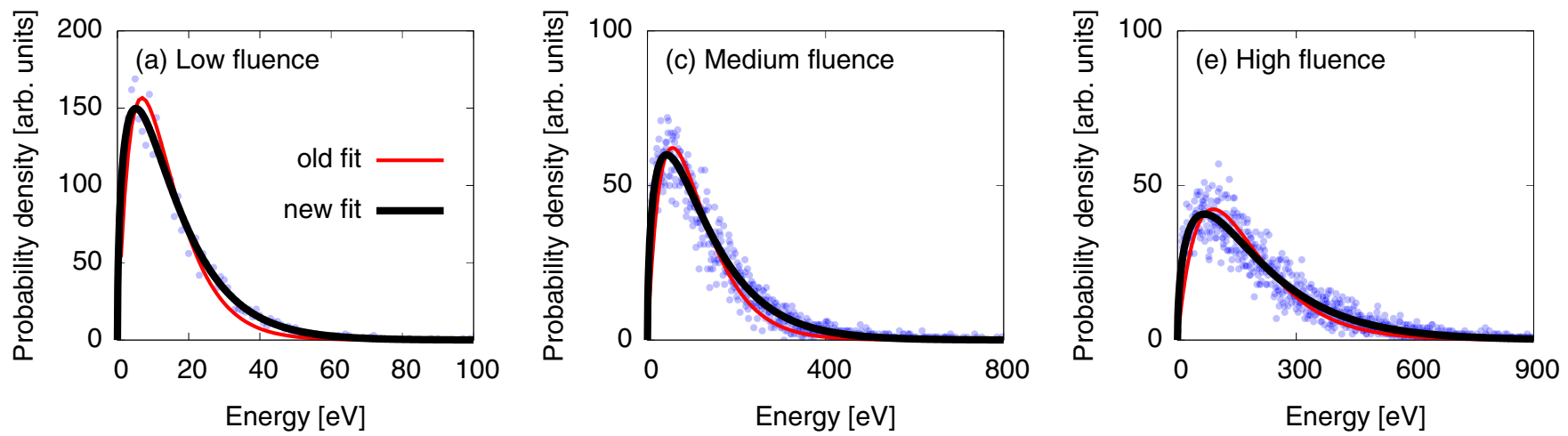

FIG. 3. Kinetic-energy distribution of the electron plasma compared with two fitted curves: The old fit is fitted to $f_{\text {wrong }}(E)$, and the new fit is fitted to $f_{E}(E)$. 